\title{
Validation of a purpose-built chewing gum and smartphone application to evaluate chewing efficiency
}

\begin{tabular}{|r|l|}
\hline Journal: & Journal of Oral Rehabilitation \\
\hline Manuscript ID & JOR-17-0402.R3 \\
\hline Danuscript Type: & Original Article \\
\hline Complete List of Authors: & $\begin{array}{l}\text { Buser, Ramona; University of Bern, Department of Reconstructive } \\
\text { Dentistry and Gerodontology } \\
\text { Ziltener, Valérie; University of Bern, Division of Gerodontology } \\
\text { Samietz, Stefanie; University Medicine Greifswald, Greifswald, Germany, } \\
\text { Department of Prosthodontics, Gerostomatology and Biomaterials, Center } \\
\text { for Oral Health } \\
\text { Fontolliet, Marta; University of Bern, Department of Reconstructive } \\
\text { Dentistry and Gerodontology } \\
\text { Nef, Tobias; University of Bern, Gerontechnology \& Rehabilitation Group; } \\
\text { University of Bern, ARTORG Center for Biomedical Engineering Research } \\
\text { Schimmel, Martin; University of Bern, Division of Gerodontology }\end{array}$ \\
\hline Patient: & geriatric, adult, adolescent \\
\hline Condition: & eating/ mastication/ swallowing/ bolus formation \\
\hline Content method: & prosthodontics \\
\hline Study method: & case control study \\
\hline &
\end{tabular}




\section{Title page}

Title: $\quad$ Validation of a purpose-built chewing gum and smartphone application to evaluate chewing efficiency

Short title: Hue-check gum

Article category: Original Research Report

Authors: $\quad$ Ramona Buser ${ }^{1^{*}}$, Valerie Ziltener $^{{ }^{*}}$, Stefanie Samietz ${ }^{2}$, Marta Fontolliet ${ }^{1}$, Tobias $\mathrm{Nef}^{3,4}$, Martin Schimmel ${ }^{1,5}$

* equal contribution as first authors

Affiliations: $\quad 1$-Department of Reconstructive Dentistry and Gerodontology, School of Dental Medicine, University of Bern, Bern, Switzerland

2 - Department of Prosthodontics, Gerostomatology and Biomaterials, Center for Oral Health, University Medicine Greifswald, Greifswald, Germany

3 - Gerontechnology \& Rehabilitation Group, University of Bern, Bern, Switzerland 4 - ARTORG Center for Biomedical Engineering Research, University of Bern, Bern, Switzerland

5 - Division of Gerodontology and Removable Prosthodontics, University Clinics of Dental Medicine, University of Geneva, Geneva, Switzerland

MeSH Terms: Mastication; Chewing Gum; Dysphagia; symptom assessment, chewing efficiency

\section{Corresponding author:}

Prof. Dr. med. dent. Martin Schimmel, MAS Oral Biol

Department of Reconstructive Dentistry and Gerodontology

Division of Gerodontology

University of Bern, School of Dental Medicine

Freiburgstrasse 7, 3010 Bern, Switzerland

martin.schimmel@zmk.unibe.ch

Tel: +4131632 2597 


\section{Abstract}

Objectives: To validate a purpose-built two-colored chewing gum (Hue-Check Gum ${ }^{\circledR}$ ), and to test the accuracy of a custom-built smartphone application for a color-mixing ability test to assess chewing function.

Methods: Fully dentate participants (28D-group) and edentulous participants with implant overdentures (IOD-group) were recruited. They chewed a Hue-Check Gum ${ }^{\circledR}$ for 5, 10, 20, 30, and 50 chewing cycles, respectively. Maximum voluntary bite force (MBF) was assessed with a digital gauge. The specimens were analyzed according to their color mixture (variance of hue, $\mathrm{VOH}$ ) with a validated software (VOH_scan) and a newly developed smartphone application (VOH_app).

Results: Participants segregated by dental state were significantly different regarding the number of occluding pairs of teeth, age, and MBF. VOH_scan showed a negative logarithmic association with the number of chewing cycles, and VOH could be predicted from chewing cycles, MBF, and gender. The clearest discrimination between the IOD and the $28 \mathrm{D}$ groups was observed for $n=20$ chewing cycles; at this point, $\mathrm{VOH}$ was distinguishably different with a p-value $<0.0001$. The $\mathrm{VOH}$ _app was a very good predictor for $\mathrm{VOH} \_s c a n$ with $0.80<$ pseudo- $\mathrm{R}^{2}<0.83$, depending on light exposure and measuring distance. There was, however, a systematic error in the accuracy of the app for inadequately chewed specimens.

Conclusions: The use of the two-colored chewing gum would provide a fast, simple, and inexpensive method for the assessment of masticatory performance, which could be used in geriatric wards or in private practices without the requirement of specialized equipment or trained staff. 


\section{Introduction}

\section{Evaluating masticatory function is important to understand oral function not only in potentially} impaired individuals, but also to evaluate the success of dental treatment. Impaired chewing function might have an influence on Quality of Life, social interaction or general health. Furthermore, chewing efficiency is a surrogate parameter for the function of many oro-facial structures, such as teeth, muscles, salivary glands and nerve receptors [1].

Assessment of masticatory function may involve subjective evaluation by the patient (masticatory ability) or objective test procedures (masticatory efficiency). The self-assessment is estimated using questionnaires or personal interviews [2]. Masticatory efficiency is the objective capacity of a person to fragment solid food and is defined as "the effort required to achieve a standardized degree of comminution" [3]. In 1902, Gaudenz was the first to describe the evaluation of chewing efficiency by implementing mechanically degradable test foods such as vegetables, fruits, meat, eggs, bread, cheese, potatoes, and maccaroni [4]. Fragmenting tests are still today regarded as the gold standard for evaluating masticatory efficiency [5].

However, fragmenting tests can be inconvenient and cost-intensive because they require specialized equipment such as sieve stacks, vibrators, drying furnaces, and other lab equipment. Furthermore, fragments of the specimens need to be retrieved completely from the oral cavity after the masticatory process, which can be very difficult with small particles. In addition, patients with dysphagia are at risk of aspirating such particles [6].

Over the last three decades, studies have investigated the feasibility of implementing alternative approaches with dysphagic patients or in geriatric homes, where no specialized lab is available. Here, a two-colored specimen made from wax or chewing gum is used, as it is easily retrievable from the oral cavity and checked for masticatory performance. Liedberg et al. described already in 1991 the use of wax to evaluate bolus kneading and formation in dysphagic patients [7]. Later developments comprised the reliable and accurate opto-electronic evaluation of chewing gums to assess two-color mixing ability, that is, bolus kneading capacity [8-11]. These tests correlate with fragmenting tests 
and can therefore be used as an objective indicator for masticatory efficiency $[12,13]$. These tests are inexpensive and can be used for research or clinical assessment of patients with a presumed impaired masticatory function [14]. However, it should be considered that in sieving tests with silicon cubes or mixing ability tests using chewing gum or wax, unnatural food products are used that cannot be swallowed and may thus create an artificial chewing pattern. Furthermore, comminution tests employing test foods like carrots show greater dependence on available bite force and may create more natural chewing patterns [5]. The bolus kneading (color-mixing) ability tests using chewing gum rely on folding motions of the tongue [15].

One challenge in the use of these tests is to find appropriate specimens, which should comply with certain requirements (Table 1 ). Often, chewing gum would stick to dentures, the taste would be inappropriate for elderly patients, or the colors would be unsuitable for analyzing the degree of color mixture with custom-made software (e.g. ViewGum ${ }^{\circledR}$, www.dhal.com) [8]. Therefore, the twocolored Hue-Check Gum ${ }^{\circledR}$ was developed for use with the two-color mixing ability test to assess masticatory performance in clinical and academic settings. The aim of the current study was to validate the newly developed two-colored Hue-Check Gum ${ }^{\circledR}$ in assessing masticatory performance. An additional goal of the study was to test the accuracy of a custom-built smartphone application (Hue-Check Gum ${ }^{\circledR}$ App $^{\circledR}$, ARTORG CENTER, University of Bern). The null hypotheses for this study were: 1) The color-mixing degree of the Hue-Check Gum ${ }^{\circledR}$ is not correlated with the number of chewing cycles and cannot be used to discriminate between different dental states. 2) The analysis of the color mixture with the Hue-Check Gum ${ }^{\circledR} \mathrm{App}^{\circledR}$ does not show significant concordance with results using the gold standard analysis method (ViewGum ${ }^{\odot}$ ).

\section{Material and Methods}

The study was conducted in compliance with the ethical principles and standards of the Declaration of Helsinki. The Bern Cantonal Ethics Committee (CEC, Req-2016-00266) authorized conducting the 
experiments without a requirement for formal approval, complying with the Swiss Human Research Act, HRA, Art. 1, §1.

\section{Inclusion criteria}

Participants representing "ideal chewers" with an intact dentition (28D-group) or edentulous subjects (IOD-group) with a presumed impaired chewing function were recruited from staff and patient pools of the School of Dental Medicine, University of Bern [16]. Participants were excluded if they showed clinical signs or history of temporomandibular disorders (TMD). Participants in the 28Dgroup were included if they were between 18 and 30 years old, have a minimum of 28 teeth, a maximum DMFT (decayed/missing/filled teeth) [17] score of 4 and an Angle class I occlusion. Participants in the IOD-group were selected from a previous study performed at the School of Dental Medicine, University of Bern [18]; they had a conventional upper denture and an overdenture in the mandible retained by four inter-foraminal narrow-diameter implants.

\section{Specimen}

The Hue-Check Gum ${ }^{\circledR}$ served as the test specimen. It is a chewing gum that was developed and produced for Orophys GmbH (Muri b. Bern, Switzerland). It is produced in compliance with Swiss and EU regulations (Good Manufacturing Practice GMP, AMBV Art. 4 Abs. 2), is sugar-free, uncoated, and has a slight mint taste. The packaging consists of two gums in dragée form, one blue and one pink, which need to be stuck together manually by wetting them with water and applying moderate force. The dimensions of the fused gums are $8 \times 20 \times 12 \mathrm{~mm}$.

Specimen hardness was evaluated utilising a Shore A-test, which is especially appropriate for soft materials. The intender, a truncated cone, is applied with a weight of $1 \mathrm{~kg}$ and subsequently evaluated according to the Shore-scale, based on insertion distance and hardness ( $0 \mathrm{~mm}=100$ Shore, $2.5 \mathrm{~mm}=0$ Shore). To evaluate consistency of the hardness over time, 10 new and 10 one-year-old specimens from each color were tested and hardness measurements subsequently compared. The 
unchewed specimens were each tested 5 times, resulting in 50 measurements for each of the four groups (pink/new, pink/old, blue/new, blue/old).

\section{Protocol}

Anamnestic parameters collected included participants' gender and age. In addition, the dental state and occlusal support were noted by counting the number of teeth, classifying according to the Eichner Index [19], and counting the number of occluding posterior units (OU), also taking denture teeth into account (1 molar equals 2 premolar units) [20]. Maximum voluntary bite force (MBF) was measured with a digital force gauge in the region of the first molar with three assessments per side (Occlusal Force-Meter GM 10 ${ }^{\circledR}$, Nagano Keiki Co., Japan). The mean of six recordings was used for further analysis.

The experiments were performed in an upright-seated position. The participants were instructed to chew five samples of gum on their preferred chewing side for $5,10,20,30$, and 50 chewing cycles respectively, leaving an interval of 1 minute between each chewing sequence. For this purpose, the specimen was positioned on their tongue with the pink side facing the palate. The operator counted the chewing cycles by observing the movement of the mandible in the anterior plane and evaluating the up-and-down movements. The specimens were then retrieved from the oral cavity and excess saliva was removed with a cotton roll or Dental Air Water Spray Triple, as available. These specimens were complemented by 10 unchewed gums that served as baseline. The gum was placed in the middle of a transparent plastic bag and then flattened to a wafer with a thickness of $1 \mathrm{~mm}$ using a custom-made polyvinyl chloride template with a milled depression of $1 \mathrm{~mm} \times 50 \mathrm{~mm} \times 50 \mathrm{~mm}$.

\section{Opto-electronic assessment}

\section{ViewGum@ Software}

As a reference analysis, the chewing efficiency was evaluated through optoelectronic analysis performed with the ViewGum ${ }^{\odot}$ software as the gold standard $[8,21]$. The samples were scanned from both sides at a resolution of 300 dpi using a flatbed scanner (Epson Perfection V750 Pro, Seiko 
Epson Corp., Japan). The software assembled the images of both sides and transformed them into the HSI color space. The operator semi-automatically segmented the image of the gum from the background, and the Variance of Hue (VOH_scan) was then calculated by the software. The operator was experienced in this analysis with very high intra-rater reliability [22].

Hue is an angle in the $\mathrm{HSI}$ color space, thus the circular variance of hue is defined as 1 minus the length of the average vector. ViewGum ${ }^{\odot}$ displays the standard deviation (SD) between the two color peaks: SD = sqrt (Variance of Hue, $\mathrm{VOH})[21]$.

The $\mathrm{VOH}$ is considered a measure of masticatory performance because it shows a logarithmic association with the number of chewing cycles [8]. A high $\mathrm{VOH}$ results from poorly mixed colors through deficient chewing, whereas adequate chewing leads to well-mixed colors and a low VOH.

\section{Hue-Check Gum ${ }^{\circledast}$ Application}

The specimens were also evaluated with the Hue-Check Gum ${ }^{\circledR}$ mobile application, developed by the ARTORG CENTER, University of Bern, Switzerland. For this, the flattened specimen wafer was placed on a white background and both sides were photographed with an iPhone 6 (8-megapixel camera resolution). These pictures were then uploaded to a dedicated server; subsequently, custom-made software automatically segmented the gums from the background and then a newly developed smartphone application ( $\left.\mathrm{VOH} \_a p p\right)$ was used to analyze the compound image of the two sides of the gum.

Each of the 210 specimens was photographed from both sides under 4 different conditions: two distances $\left(D_{1}=10 \mathrm{~cm}\right.$; the outline of the gum should fit exactly the center circle of the camera image, $\left.D_{2}=30 \mathrm{~cm}\right)$ and two different lighting conditions ( $L_{1}=$ lower brightness, $L_{2}=$ higher brightness $)$. Distances were verified with a mounted ruler, but pictures were taken freehand to simulate realistic clinical applications. The light conditions were standardized with mounted desk lights; however, the luminous flux of the light was not objectively measured. 


\section{Statistical analysis}

All numerical variables were analyzed descriptively by computing means, standard deviations, medians, and inter-quartile ranges, as appropriate. VOH_scan was analyzed with linear mixed-effects models, taking the repeated measurements into account. Basic and multiple regression models were performed for both groups separately. Model diagnostics were performed by drawing normal QQ plots and performing a Shapiro-Wilk normality test.

The agreement between the gold standard assessment (VOH_scan) and the mobile application (VOH_app) was tested with Bland-Altman plots [23]. A decision of the best predictor of VOH_app to VOH_scan with respect to the conditions of assessment $L_{1} D_{1}, L_{2} D_{1}, L_{1} D_{2}$, and $L_{2} D_{2}$ was based on the pseudo- $\mathrm{R}^{2}$ among the four linear mixed-effects models using the $\mathrm{VOH}$ _app as a fixed-effect predictor and $\mathrm{VOH} \_s c a n$ as the dependent variable. Group comparisons were performed with two-sample Student's t tests for samples with unequal variances. Comparison of hardness was performed using Mann-Whitney $U$ tests. The sample size estimation was based on previous experiments [8] to achieve a power of $90 \%$; the significance threshold was set to $5 \%$.

All statistical results were calculated with R 3.2.2 (R Project for Statistical Computing, Vienna, Austria) by a senior biostatistician.

\section{Results}

\section{Participants}

Each group included 20 participants. The participants of the 28D-group were $24.8 \pm 8.2$ years old (mean \pm s.d., 7 women, 13 men); the IOD-Group participants were $72.6 \pm 8.2$ years old ( 15 female, 5 male) (age: $p<0.0001$ ). The mean number of occluding units (OU) in the 28D- and IOD-Groups was $12.1 \pm 0.4$, and $8.0 \pm 0$, respectively $(p<0.0001)$. MBF was also significantly different between groups (IOD-group 152.4 $\pm 82.1 \mathrm{~N}, 28 \mathrm{D}$-group $555.8 \pm 230.2 \mathrm{~N}, \mathrm{p}<0.0001$ ). 


\section{Specimens}

The mean Shore hardness of the new pink gum was $32.2 \pm 4.2$, and of the one-year-old specimens $30.6 \pm 7.7(p=0.5967)$. The new blue specimens had a Shore hardness of $21.7 \pm 5.2$, and the oneyear old gums $25.2 \pm 6.4(p=0.1124)$.

\section{Validation Hue Check Gum ${ }^{\circledR}$}

Hue-Check Gum ${ }^{\circledast}$ showed significantly different color-mixing characteristics depending on the number of chewing cycles. The $\mathrm{VOH} \_s c a n$ decreased with an increasing number of chewing cycles and could be predicted from the number of chewing cycles in a simple fixed effect regression model (28D-group: estimate -0.1422, standard error 0.0081, $\mathrm{p}<0.0001$; IOD-group: estimate -0.1650, standard error $0.0101, \mathrm{p}<0.0001)$

For the 28D-group, chewing efficiency showed a negative logarithmic association with the number of chewing cycles. In the IOD-group, this parameter showed a more linear negative association with a very large inter-individual variability (Figs. 1a, 1b). The standard deviations were greater within the different cycle counts for the IOD-group than for the 28D-group; the best discrimination between groups was observed at $\mathrm{n}=20$ cycles. The difference in $\mathrm{VOH}$ _scan between the two groups at $\mathrm{n}=20$ cycles was highly significant ( $p<0.0001)$ (Fig. 2).

\section{Predictors of chewing performance}

The multiple mixed-effects models with fixed effects revealed that VOH could be predicted from the number of chewing cycles, MBF, and gender. There was a statistical tendency for age as a predictor (Table 2). With the applied statistical model, no effects were calculated for the number of teeth and $\mathrm{OU}$, as the variations were too small.

\section{Validation Hue-Check Gum ${ }^{\circledR}$ Application}

The assessment with the smartphone application $V O H_{-}$app for the different settings $\left(L_{1} D_{1}, L_{2} D_{1}\right.$, $L_{1} D_{2}$, and $L_{2} D_{2}$ ) showed a good agreement with the gold standard VOH_scan according to the Bland- 
Altmann analysis. The differences between $\mathrm{VOH}$ _app and $\mathrm{VOH}$ _scan became larger with increasing VOH values. There was a systematic difference around the mean VOH of 0.6 in all conditions (Fig. 3). To identify the best possible predictor for VOH_scan, pseudo- $\mathrm{R}^{2}$ values were computed from linear mixed-effects models for each condition $L_{1} D_{1}, L_{2} D_{1}, L_{1} D_{2}$, and $L_{2} D_{2}$ as a measurement of goodness-offit likelihood. This test revealed very similar and high pseudo- $\mathrm{R}^{2}$ values indicating VOH_app as a good predictor for all conditions of measurement (Table 3). The absolute and relative mean error was smallest for $L_{2} D_{1}$ among assessed conditions and therefore was considered the best predictor for VOH_scan.

\section{Discussion}

\section{Summary of the results}

The current study demonstrated that the individual color-mixing ability and therefore masticatory performance can be evaluated with an opto-electronic analysis of the purpose-built Hue-Check Gum $^{\circledR}$. The applied method can discriminate between subjects with different dental states, especially after chewing the test gum for 20 chewing cycles. Hence, null hypothesis 1 must be rejected.

The analysis of the degree of color mixture with a smartphone application is a very good predictor of chewing efficiency as assessed with the gold standard ViewGum ${ }^{\odot}$, especially when used under conditions of bright lighting and at a distance of $10 \mathrm{~cm}$. Therefore, null hypothesis 2 must also be rejected.

\section{Strengths and weaknesses of the study}

Chewing is a very complex function of the oro-facial system and several factors play an important role to comminute foodstuff and form a bolus that is safe to swallow. The most important predictor for chewing efficiency might be the number of occluding pairs of teeth, but the maximum available force, saliva flow rate, prosthetic reconstruction, force and coordination of tongue and cheeks, age, and gender also play important roles [24]. Cognitive state and intra-oral sensitivity are also 
associated with chewing function $[25,26]$. Taking all these confounders into account is barely possible. In the current study, age, gender, OU, and MBF were evaluated and served as predictors in the statistical models.

\section{Test food}

There is evidence that comminution tests might depend more on the maximum available jaw closing force than color-mixing ability tests that employ plastic-elastic specimens, which continue to soften during the chewing sequence. If the gum is too soft, MBF will only show significant variation when the physiological spare capacity is reduced [8]. The Hue-Check Gum ${ }^{\circledast}$, however, seems to be hard enough to take this factor into account.

Various test foods can be used to evaluate masticatory performance, with each having distinct physiological characteristics. Natural food simulates a natural chewing act but may trigger the swallowing reflex. Furthermore, natural food can seldom be preserved over a long time and its storage can be sensitive. In contrast, artificial material such as silicone leads to a more conscious than natural act of chewing but can be stored easily and over a long period of time. Chewing gum is considered as artificial material, with numerous advantages: it is easily available, its consistency is reproducible, it is inexpensive, and can be easily stocked and stored long-term without changing its character. This study confirms the final point, since the change in hardness of the tested specimens after one year of storage was not statistically significant. Previous studies have used and tested several types of chewing gum or wax $[8-10,12,27,28]$, but they are either no longer available, contain sugar, or stick to dentures and therefore unsuitable for bolus-kneading tests. Finding an appropriate specimen for bolus-kneading tests was a central objective of this study. HueCheck Gum ${ }^{\circledast}$ fulfilled most of the demanded criteria (Table 1). Inter alia, it is sugar-free, does not stick to denture material, is amenable to long-term storage, and is easily available. In addition, the color combination is ideal for analysis in the selected color space, as it represents a large spread in Hue-values [21]. 


\section{Subjects}

Participants in both groups were recruited based on specific criteria. An ongoing study evaluating narrow-diameter implants in edentulous patients [18] allowed us to recruit participants with presumed impaired chewing function and to compare them to "ideal" chewers [16].

One objective of this study was to find the ideal number of chewing cycles for this specific chewing gum. Chewing gum has a complex rheological and mechanical behavior, as it becomes softer and smooth during the chewing process. There are only a few gum bases, but the addition of essential oils (e.g. peppermint oil) will soften them and give each chewing gum a different set of mechanical properties. Hence, each gum must be individually assessed and validated for color-mixing ability tests. Chewing cycles in this study were chosen to cover the full extent of possible color-mixing degrees. The mean curves indicate that 20 chewing cycles should be used for the bolus-kneading test. This number best discriminates masticatory performance between an ideally-chewing, fully dentate individual (28D-group) and an edentulous person with impaired chewing function (IODgroup). Similar to previous studies, 20 chewing cycles seemed to be ideal, but other gums might require varying numbers of chewing cycles [29]. So far, several mixing-ability tests have come to the same conclusion about the use of this number of chewing cycle [8-10, 12, 27].

The loss of all teeth leads to accelerated muscle wasting and accentuates the age-dependent atrophy of the jaw-closing muscles [30]. There is evidence that MBF remains the same even with increasing age if the dental state remains unchanged $[24,31]$. However, there is a distinct difference in MBF if fewer teeth are present $[22,32]$. In the current study, we observe a non-significant tendency for age to be a predictor for chewing efficiency. Hence, age itself seems to have a smaller influence than the fact that all teeth were replaced in the IOD group [33].

The current study also reveals some interesting findings about the rehabilitation of oral function by means of oral implants in edentulous subjects. It seems intuitive to claim that stabilization with dental implants will automatically improve chewing efficiency, and a recent review aimed to provide evidence for this claim. The authors concluded that "treating complete denture wearers with 
implants to support their denture improves their chewing efficiency, increases maximum bite force and clearly improves satisfaction" [34]. However, looking closely at the evidence, the picture becomes more complex and may depend on the instruments used for the assessment. Studies employing comminution tests rely largely on the maximum available bite force, as the test food must be hard and breakable (e.g. nuts or silicone cubes); usually, the stabilization of dentures with implants will reliably increase the maximum bite force. Hence, these tests show an improved masticatory efficiency in edentulous subjects with implants relative to conventionally restored patients $[35,36]$.

However, studies employing mixing ability tests show a different picture. These tests rely less on the crushing of the food, but more on the bolus forming and kneading. These tests are not as dependent on bite force, as the specimens are deformable and often soft, but rather on the force, coordination, and sensitivity of the soft tissues (e.g. the tongue, palate, and cheeks) that are affected by aging itself $[26,37]$. Of course, the structures are less, if at all, affected by any implant therapy designed to stabilize the denture; consequently, these tests often fail to demonstrate a short-term (up to 1 year) effect on chewing efficiency $[18,22,32,38]$.

A very interesting train of thought was recently discussed by a group at the University in ClermontFerrand, which measured chewing efficiency in eleven patients before and after stabilizing the lower denture with four narrow-diameter implants. They clearly showed that some implant patients benefit from such treatment, whereas other remain severely impaired, even with contemporary oral rehabilitation procedures [39]. Our standard statistical tests might fail when analyzing the complex and highly variable development in oral function after invasive procedures, e.g. implant placement (Fig 1a). Even with dental implants, edentulous patients remain severely impaired, as many of the intra-oral receptors for guiding the force and movement of the mandible have been lost. Clinicians should adopt a patient-level perspective and aim to develop predictors prior to invasive implant therapy, whether a patient will benefit from the treatment or not. 
To rigorously assess the impact of any dental procedure or oral impairment on a patient's ability to form a bolus that is safe to swallow, subjective assessments should be also employed, such as evaluating food avoidance or swallowing unchewed foodstuff [40].

\section{Results in the context of existing knowledge}

Some studies have demonstrated a significant correlation between the color-mixing and fragmenting tests $[12,13]$. It was demonstrated that color-mixing tests based on bolus kneading better discriminate between subjects with impaired chewing function than fragmenting tests [12]. However, the color-mixing test is less applicable for young subjects with good masticatory performance. Therefore, it could be concluded that color-mixing tests to evaluate the masticatory performance based on bolus-kneading are most suitable for elderly subjects with compromised chewing function. Unlike the food particles produced during the fragmenting test, chewing gum can be retrieved easily and entirely from the patients' oral cavity with a low aspiration risk.

\section{Clinical or other practical implications}

It has been demonstrated that two-colored bolus-kneading tests can be used as an alternative to the current gold standard methods, and can be employed in clinical research settings to indirectly assess masticatory function $[13,18,26,32,38,41]$. The described bolus-kneading test with the use of the Hue-Check Gum ${ }^{\circledast}$ allows a fast and simple application of the color-mixing method. It is time-saving and does not require specialized and expensive equipment or trained professionals. Furthermore, the test does not cause great discomfort for the patient, takes less than 1 minute of his/her time, and the specimen can be retrieved fast and easily in toto, unlike the fragmenting tests, where the patient needs to spit several times, which can be very unpleasant.

To facilitate even greater ease-of-use for the bolus-kneading test, an application for smartphones was developed. Using this application, the need for a scanner and a computer to analyze the flattened specimen can be eliminated, which would make sample evaluation significantly easier and more straightforward in settings such as elderly homes. The results were very promising, especially 
when pictures were taken under conditions of bright lighting and a distance of $\approx 10 \mathrm{~cm}$. We believe that with further development, the application could quite easily be improved, eradicating the systematic error at the $\mathrm{VOH}$ mean of 0.6. Unfortunately, further development of the application is unlikely for administrative reasons: since the results from the application can be used for diagnostic and therapeutic decisions, it is considered a class lla medical device according to the European regulations of medical devices (MDR). This classification requires a certified external quality management that would make the approach extremely financially straining.

\section{Suggestions for further research}

Further research should aim to demonstrate causal relationships between masticatory function and general conditions such as nutritional state or cognitive function [42]. There are numerous validated and suitable tests currently available to easily assess masticatory function in clinical and scientific settings. The test described here could even be applied in elderly homes or hospitals to elucidate the importance of masticatory function on quality of life and well-being. Because chewing efficiency is a compound and functional measure of the oro-facial system, the test could eventually replace other diagnostics like tooth counting or bite force assessment in epidemiological studies.

\section{Summary and Conclusions}

Mastication and swallowing are among the most complex functions of the oro-facial system. The evaluation of masticatory performance with a color-mixing ability test is a compound assessment of almost all structures and functions involved in forming a safe-to-swallow food bolus. The Hue-Check Gum $^{\circledR}$ is a valid and suitable specimen, fulfilling most of the requirements for application in such tests.

Color mixture may be assessed with PC-based software, but a specially designed smartphone application also produces valid results for evaluating chewing efficiency in conjunction with the HueCheck Gum ${ }^{\circledast}$. This approach would provide a fast, simple, and inexpensive method for the 
assessment of masticatory performance that could be used in geriatric wards or private practices without the requirement of specialized equipment or trained staff.

\section{Acknowledgments}

Conflict of interest: Martin Schimmel is co-founder of Orophys Gmbh (Muri b. Bern, Switzerland). All other authors declare no conflict of interest. Funding by private funds of the Division of Gerodontology, and the ARTORG Center, University of Bern, Switzerland. The specimens were provided by Orophys GmbH, Switzerland, without benefit in cash or kind. Dr. K. Memedi, University of Bern performed the analysis of $\mathrm{VOH} \_$scan. Mr. A. Mitache was involved in the development of the smartphone application. Statistical analysis was performed by Gabriel Fischer, MSc (significantis GmbH, Niederwangen b. Bern, Switzerland). We thank Alexandra Quooß (Dipl. Ing. (FH) dental technologies, Greifswald, Germany) for technical support with the Shore A-test. Language revision by Dr. rer. nat. John Michael Lindner (Freiburg, Germany). 


\section{Figure legends}

\section{Figure $1 \mathrm{a}$ and Figure $1 \mathrm{~b}$}

Line graphs for each individual participant showing chewing efficiency as assessed with the variance of hue $(\mathrm{VOH})$ measurements by the gold-standard software ViewGum $\odot$, both for the group of edentulous participants receiving stabilization of the lower denture with two implants and unsplinted attachments (IOD-Group, Fig 1a) and the group of dentate participants (28D-group, Fib 1b). The graphs visualize the large inter-individual variability with respect to masticatory function, even when patients were provided with contemporary oral rehabilitation procedures.

\section{Figure 2}

Mean curves with standard deviations of the variance of hue $(\mathrm{VOH})$ according to various dental states and the number of chewing cycles as calculated by the gold-standard software ViewGum@. IOD-Group, cohort of edentulous participants; 28D-Group, cohort with complete dentition.

\section{Figure 3}

Bland-Altman-Plots comparing the four settings $L_{1} D_{1}, L_{2} D_{1}, L_{1} D_{2}$, and $L_{2} D_{2}$ for the smartphone application versus evaluation of color-mixture with the gold standard PC-based software (ViewGum(C). VOH, variance of hue, number of chewing cycles $n=20$. 


\section{Tables}

\section{Table 1}

Specifications for an ideal specimen in a bolus-kneading test employing color-mixing analysis [8].

1. The specimen should have two colors, ideally pre-combined in one piece.

2. The color combination should represent a large spread in hue values in the HIS color space (e.g. green/red or red/azure).

3. The colors should not include white, which has an undefined hue value.

4. The colors should both be visible in the unchewed gum, ideally one color per side (a colored "core" is unsuitable).

5. The specimen should not stick to denture resin (PMMA).

6. The specimen should not be too big or too hard, thus relatively easy to chew.

7. The specimen should be storable and be widely available.

8. The specimen should be individually packed for handling and hygienic reasons.

9. The colors should be relatively stable over time, even once the specimen has been chewed.

10. The taste should be enjoyable for most patients.

11. The specimen should be sugar-free. 
Table 2

Regression table for the multiple mixed-effects model with fixed effects for the dentate group (28Dgroup) and the overdenture group (IOD-group) to predict masticatory performance (VOH) from the number of chewing cycles (cycles), maximum voluntary bite force (MBF), age (years), and gender.

\section{IOD-group}

\begin{tabular}{llll}
\hline & coefficient & Standard Error & $p$-value \\
\hline Intercept & 0.7425 & 0.2604 & 0.0044 \\
Cycles & -0.1650 & 0.0101 & $<0.0001$ \\
MBF & -0.0012 & 0.0003 & 0.0006 \\
Age & 0.0055 & 0.0031 & 0.0778 \\
Gender & 0.1358 & 0.0545 & 0.0127 \\
\hline 28D-group & & & $p-$ value \\
\hline & coefficient & Standard Error & 0.0914 \\
Intercept & 1.0490 & 0.6215 & $<0.0001$ \\
Cycles & -0.1422 & 0.0079 & 0.0118 \\
MBF & -0.0002 & 0.0001 & 0.0699 \\
Age & -0.0139 & 0.0077 & 0.0395 \\
Gender & -0.0535 & 0.0260 &
\end{tabular}




\section{Table 3}

Pseudo- $R^{2}$ and mean errors for the 4 pairs of measurement conditions for the smartphone application. $\left(D_{1}=10 \mathrm{~cm}\right.$, the outline of the gum should fit exactly within the center circle of the camera image; $D_{2}=30 \mathrm{~cm} ; L_{1}=$ lower brightness; $L_{2}=$ higher brightness).

\begin{tabular}{llll} 
Test condition & Pseudo- $R^{2}$ & (absolute) mean error & (relative) mean error \\
\hline $\mathrm{L}_{1} \mathrm{D}_{1}$ & 0.80 & $-2.7331 \mathrm{e}-16$ & $-7.61110 \mathrm{e}-16$ \\
$\mathrm{~L}_{1} \mathrm{D}_{2}$ & 0.81 & $-4.3724 \mathrm{e}-16$ & $-1.21762 \mathrm{e}-15$ \\
$\mathrm{~L}_{2} \mathrm{D}_{1}$ & 0.81 & $-3.3000 \mathrm{e}-19$ & $-9.10000 \mathrm{e}-19$ \\
$\mathrm{~L}_{2} \mathrm{D}_{2}$ & 0.83 & $-5.3337 \mathrm{e}-16$ & $-1.48534 \mathrm{e}-15$ \\
\hline
\end{tabular}




\section{References}

[1] Chen J, Engelen L. Food Oral Processing: Fundamentals of Eating and Sensory Perception: Wiley-Blackwel; 2012.

[2] Boretti G, Bickel M, Geering A H. A Review of Masticatory Ability and Efficiency. J Prosthet Dent. 1995;74: 400-3.

[3] TheAcademyofProsthodontics. The Glossary of Prosthodontic Terms: Ninth Edition. J Prosthet Dent. 2017;117: e1-e105.

[4] Gaudenz J. Ueber die Zerkleinerung und Lösung von Nahrungsmitteln beim Kauact. Arch $f$ Hyg. 1902;39: 230-51.

[5] Woda A, Nicolas E, Mishellany-Dutour A, Hennequin M, Mazille M N, Veyrune J Let al. The masticatory normative indicator. J Dent Res. 2010;89: 281-5.

[6] Schimmel M, Leemann B, Herrmann F R, Kiliaridis S, Schnider A, Müller F. Masticatory function and bite force in stroke patients. J Dent Res. 2011;90: 230-4.

[7] Liedberg B, Owall B. Masticatory Ability in Experimentally Induced Xerostomia. Dysphagia. 1991;6: 211-3.

[8] Schimmel M, Christou P, Miyazaki H, Halazonetis D, Herrmann F R, Müller F. A novel colourimetric technique to assess chewing function using two-coloured specimens: Validation and application. J Dent. 2015;43: 955-64.

[9] Prinz J, F. Quantitative evaluation of the effect of bolus size and number of chewing strokes on the intra-oral mixing of a two-colour chewing gum. J Oral Rehabil. 1999;26: 243-7.

[10] Schimmel M, Christou P, Herrmann F, Müller F. A two-colour chewing gum test for masticatory efficiency: development of different assessment methods. J Oral Rehabil. 2007;34: 6718.

[11] Hayakawa I, Watanabe I, Shigezo H, Masanori N, Tetsuya S. A Simple Method for Evaluating Masticatory Performance Using a Color-Changeable Chewing Gum. Int J Prosthodont. 1998;11: 1736.

[12] Speksnijder C M, Abbink J H, van der Glas H W, Janssen N G, van der Bilt A. Mixing ability test compared with a comminution test in persons with normal and compromised masticatory performance. Eur J Oral Sci. 2009;117: 580-6.

[13] Kaya M S, Guclu B, Schimmel M, Akyuz S. Two-color chewing gum mixing ability test for evaluating masticatory performance in children with mixed dentition: Validity and reliability study. J Oral Rehabil. 2017;44: 827-34.

[14] Schimmel M, Katsoulis J, Genton L, Müller F. Masticatory function and nutrition in old age. Swiss Dent J. 2015;125: 449-54.

[15] Yamada A, Kanazawa M, Komagamine Y, Minakuchi S. Association between tongue and lip functions and masticatory performance in young dentate adults. J Oral Rehabil. 2015;42: 833-9.

[16] Fontijn-Tekamp F A, Slagter A P, Van Der Bilt A, Van 'T Hof M A, Witter D J, Kalk Wet al. Biting and chewing in overdentures, full dentures, and natural dentitions. J Dent Res. 2000;79: 1519-24.

[17] Petersen P E, Baez R J, WHO. Oral health surveys : basic methods - 5th ed. 5 edn ed. Geneva: WHO Press; 2013.

[18] Enkling N, Saftig M, Worni A, Mericske-Stern R, Schimmel M. Chewing Efficiency, Bite Force and Oral Health-Related Quality of Life with Narrow Diameter Implants - A Prospective Clinical Study: Results after One Year. Clin Oral Implants Res. 2017;28: 476-82.

[19] Eichner K. [Renewed examination of the group classification of partially edentulous arches by Eichner and application advices for studies on morbidity statistics]. Stomatol DDR. 1990;40: 321-5.

[20] Kayser A F. Shortened dental arches and oral function. J Oral Rehabil. 1981;8: 457-62.

[21] Halazonetis D J, Schimmel M, Antonarakis G S, Christou P. Novel software for quantitative evaluation and graphical representation of masticatory efficiency. J Oral Rehabil. 2013;40: 329-35. 
[22] Schimmel M, Memedi K, Parga T, Katsoulis J, Muller F. Masticatory Performance and Maximum Bite and Lip Force Depend on the Type of Prosthesis. Int J Prosthodont. 2017;30: 565-72.

[23] Bland J M, Altman D G. Agreement between methods of measurement with multiple observations per individual. J Biopharm Stat. 2007;17: 571-82.

[24] Ikebe K, Matsuda K, Morii K, Furuya-Yoshinaka M, Nokubi T, Renner R P. Association of masticatory performance with age, posterior occlusal contacts, occlusal force, and salivary flow in older adults. Int J Prosthodont. 2006;19: 475-81.

[25] Weijenberg R A, Lobbezoo F, Visscher C M, Scherder E J. Oral mixing ability and cognition in elderly persons with dementia: a cross-sectional study. J Oral Rehabil. 2015;42: 481-6.

[26] Schimmel M, Voegeli G, Duvernay E, Leemann B, Muller F. Oral tactile sensitivity and masticatory performance are impaired in stroke patients. J Oral Rehabil. 2017;44: 163-77.

[27] Endo T, Komatsuzaki A, Kurokawa H, Tanaka S, Kobayashi Y, Kojima K. A two-colored chewing gum test for assessing masticatory performance: a preliminary study. Odontology. 2014;102: 68-75.

[28] Liedberg B, Owall B. Oral bolus kneading and shaping measured with chewing gum. Dysphagia. 1995;10: 101-6.

[29] Silva L, Nogueira T, Rios L, Schimmel M, Leles C. Reliabilty of a two-colour chewing gum test to assess masticatory performance in complete denture wearers. J Oral Rehabil. 2018;45: 301-7.

[30] Newton J, Yemm R, Abel R, Menhinick S. Changes in human jaw muscles with age and dental state. Gerodontology. 1993;10: 16-22.

[31] van der Bilt A, Burgers M, van Kampen F M, Cune M S. Mandibular implant-supported overdentures and oral function. Clin Oral Implants Res. 2010;21: 1209-13.

[32] Müller F, Hernandez M, Grutter L, Aracil-Kessler L, Weingart D, Schimmel M. Masseter muscle thickness, chewing efficiency and bite force in edentulous patients with fixed and removable implant-supported prostheses: a cross-sectional multicenter study. Clinical Oral Implants Research. 2012;23: 144-50.

[33] Kumar A, Kothari M, Grigoriadis A, Trulsson M, Svensson P. Bite or brain: Implication of sensorimotor regulation and neuroplasticity in oral rehabilitation procedures. J Oral Rehabil. 2018;45: 323-33.

[34] Boven G C, Raghoebar G M, Vissink A, Meijer H J. Improving masticatory performance, bite force, nutritional state and patient's satisfaction with implant overdentures: a systematic review of the literature. J Oral Rehabil. 2015;42: 220-33.

[35] Giannakopoulos N N, Corteville F, Kappel S, Rammelsberg P, Schindler H J, Eberhard L. Functional adaptation of the masticatory system to implant-supported mandibular overdentures. Clin Oral Implants Res. 2017;28: 529-34.

[36] van der Bilt A, Van Kampen F M C, Cune M S. Masticatory function with mandibular implantsupported overdentures fitted with different attachment types. Eur J Oral Sci. 2006;114: 191-6.

[37] Machida N, Tohara H, Hara K, Kumakura A, Wakasugi Y, Nakane Aet al. Effects of aging and sarcopenia on tongue pressure and jaw-opening force. Geriatr Gerontol Int. 2016.

[38] Müller F, Duvernay E, Loup A, Vazquez L, Herrmann F R, Schimmel M. Implant-supported mandibular overdentures in very old adults: a randomized controlled trial. J Dent Res. 2013;92: 154S$60 \mathrm{~S}$.

[39] Batisse C, Bonnet G, Bessadet M, Veyrune J L, Hennequin M, Peyron M Aet al. Stabilization of mandibular complete dentures by four mini implants: Impact on masticatory function. J Dent. 2016;50: 43-50.

[40] Woda A, Hennequin M, Peyron M A. Mastication in humans: finding a rationale. J Oral Rehabil. 2011;38: 781-4.

[41] Elsig F, Schimmel M, Duvernay E, Giannelli S V, Graf C E, Carlier Set al. Tooth loss, chewing efficiency and cognitive impairment in geriatric patients. Gerodontology. 2015;32: 149-56.

[42] Ohkubo C, Morokuma M, Yoneyama Y, Matsuda R, Lee J S. Interactions between occlusion and human brain function activities. J Oral Rehabil. 2013;40: 119-29. 


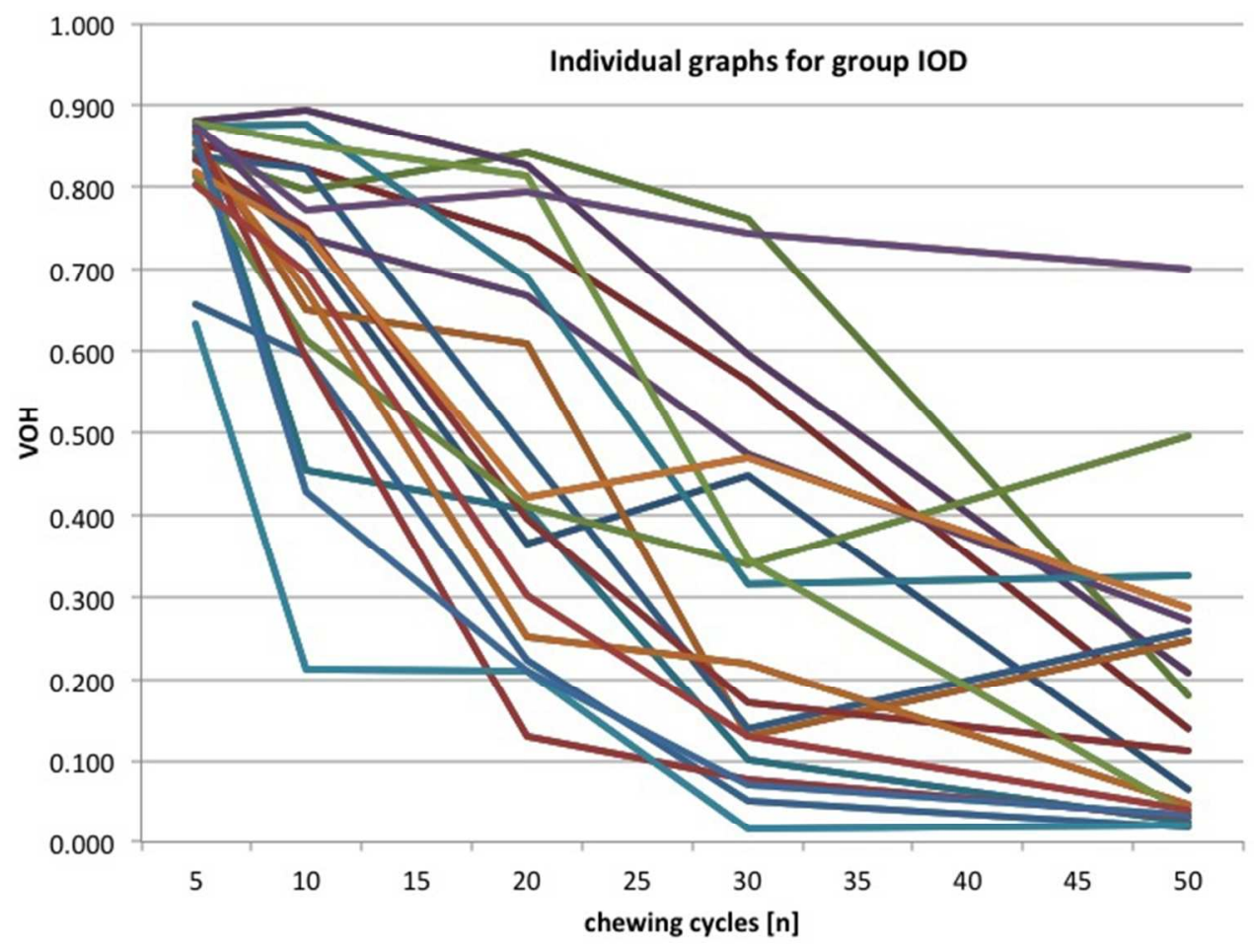

Fig 1a Line graphs for each individual participant showing chewing efficiency as assessed with the variance of hue (VOH) measurements by the gold-standard software ViewGum (c), both for the group of edentulous participants receiving stabilization of the lower denture with two implants and unsplinted attachments (IODGroup, Fig 1a) and the group of dentate participants (28D-group, Fib 1b). The graphs visualize the large inter-individual variability with respect to masticatory function, even when patients were provided with contemporary oral rehabilitation procedures.

$254 \times 190 \mathrm{~mm}(72 \times 72 \mathrm{DPI})$ 


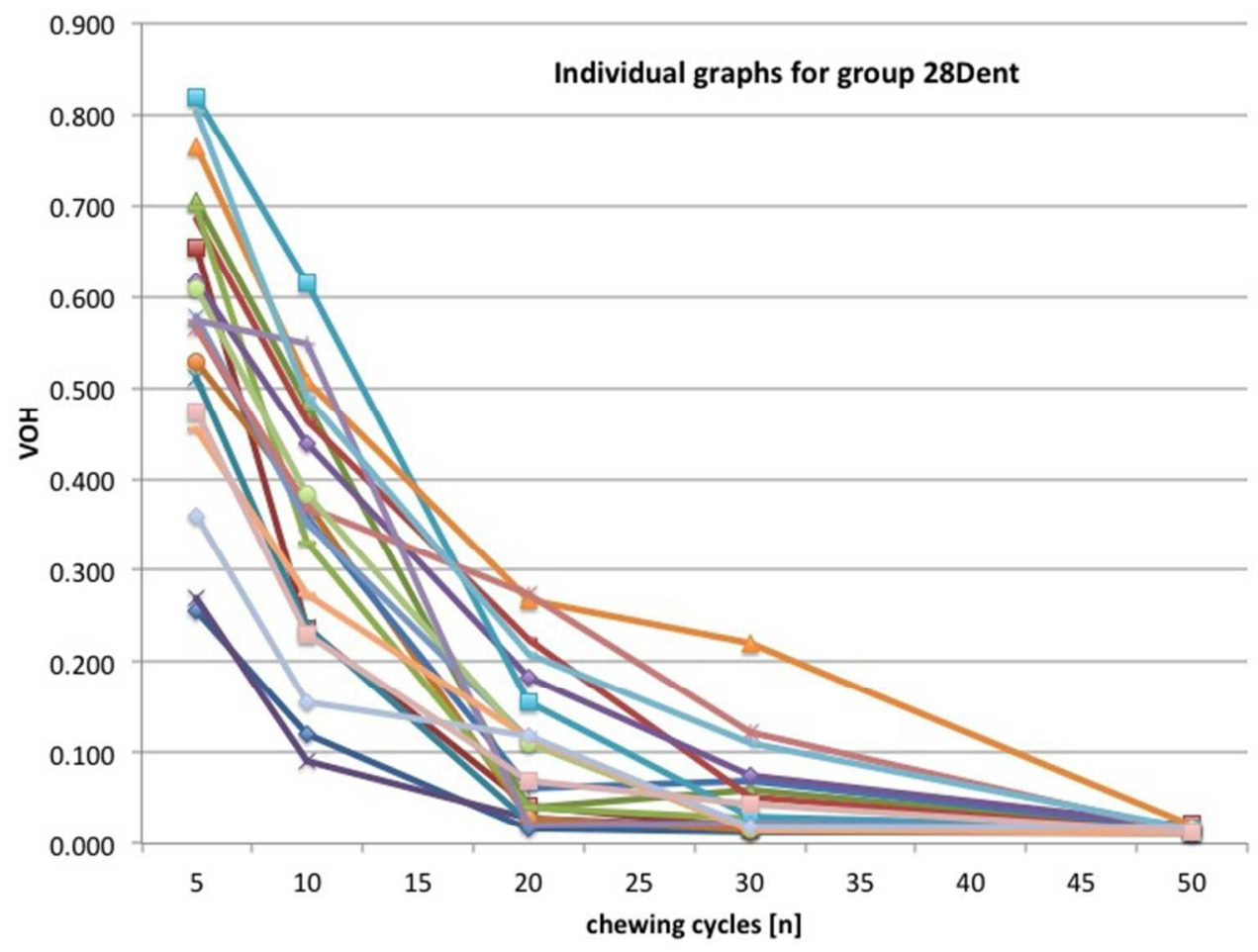

Fig 1b Line graphs for each individual participant showing chewing efficiency as assessed with the variance of hue (VOH) measurements by the gold-standard software ViewGum (c), both for the group of edentulous participants receiving stabilization of the lower denture with two implants and unsplinted attachments (IODGroup, Fig 1a) and the group of dentate participants (28D-group, Fib 1b). The graphs visualize the large inter-individual variability with respect to masticatory function, even when patients were provided with contemporary oral rehabilitation procedures.

$254 \times 190 \mathrm{~mm}(72 \times 72 \mathrm{DPI})$ 


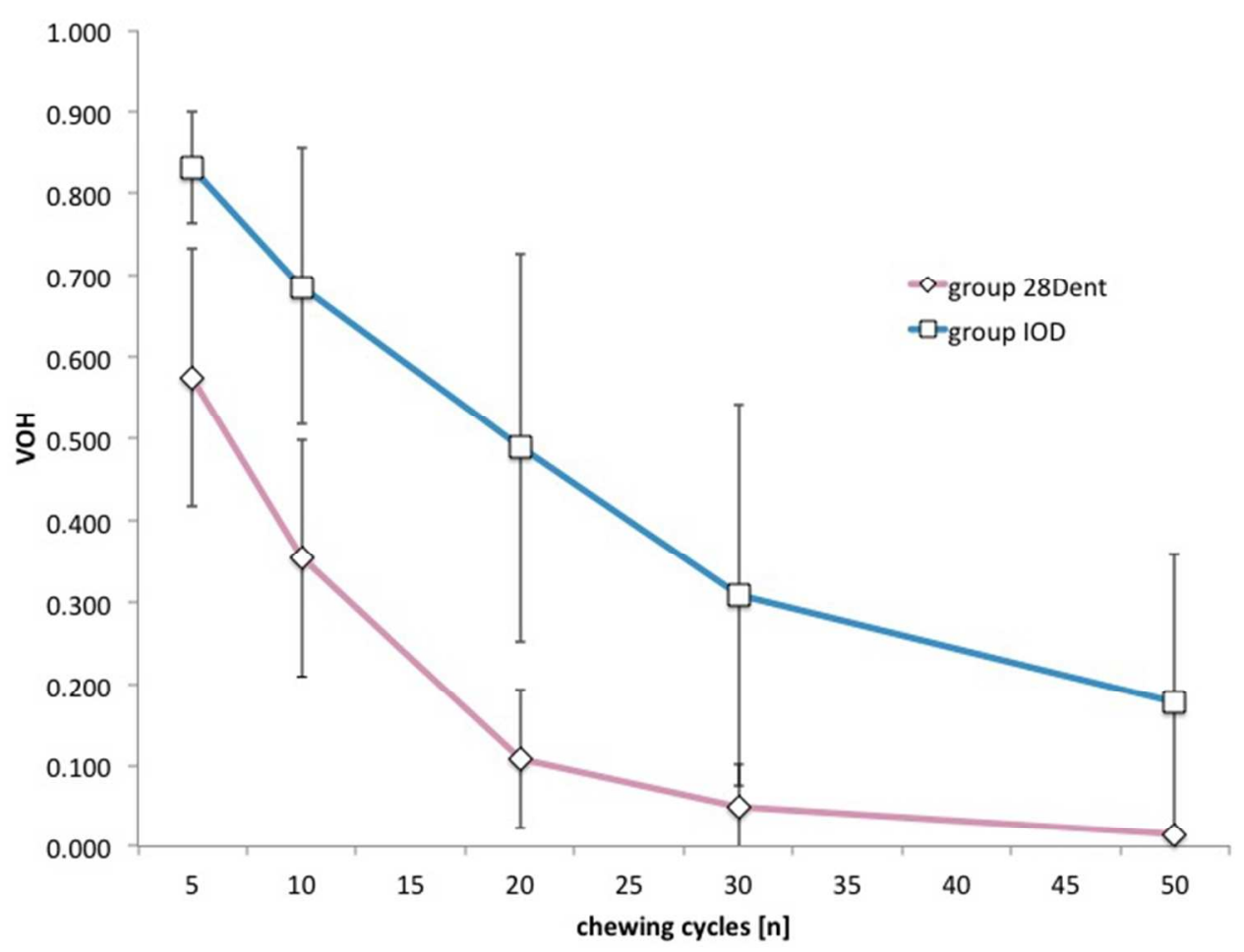

Mean curves with standard deviations of the variance of hue $(\mathrm{VOH})$ according to various dental states and the number of chewing cycles as calculated by the gold-standard software ViewGum@. IOD-Group, cohort of edentulous participants; 28D-Group, cohort with complete dentition.

$254 \times 190 \mathrm{~mm}(72 \times 72 \mathrm{DPI})$ 

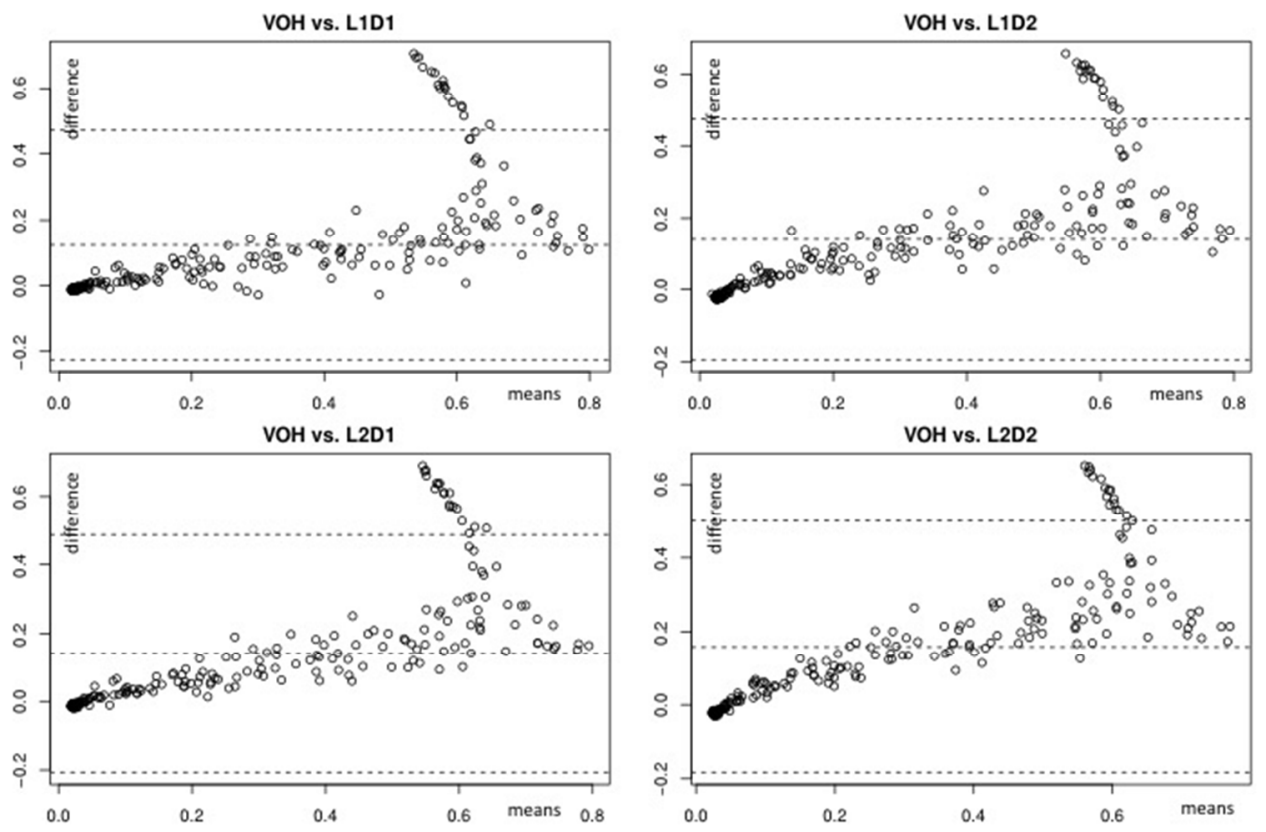

Bland-Altman-Plots comparing the four settings L1D1, L2D1, L1D2, and L2D2 for the smartphone application versus evaluation of color-mixture with the gold standard PC-based software (ViewGum@). VOH, variance of hue, number of chewing cycles $n=20$.

$254 \times 190 \mathrm{~mm}(72 \times 72 \mathrm{DPI})$ 


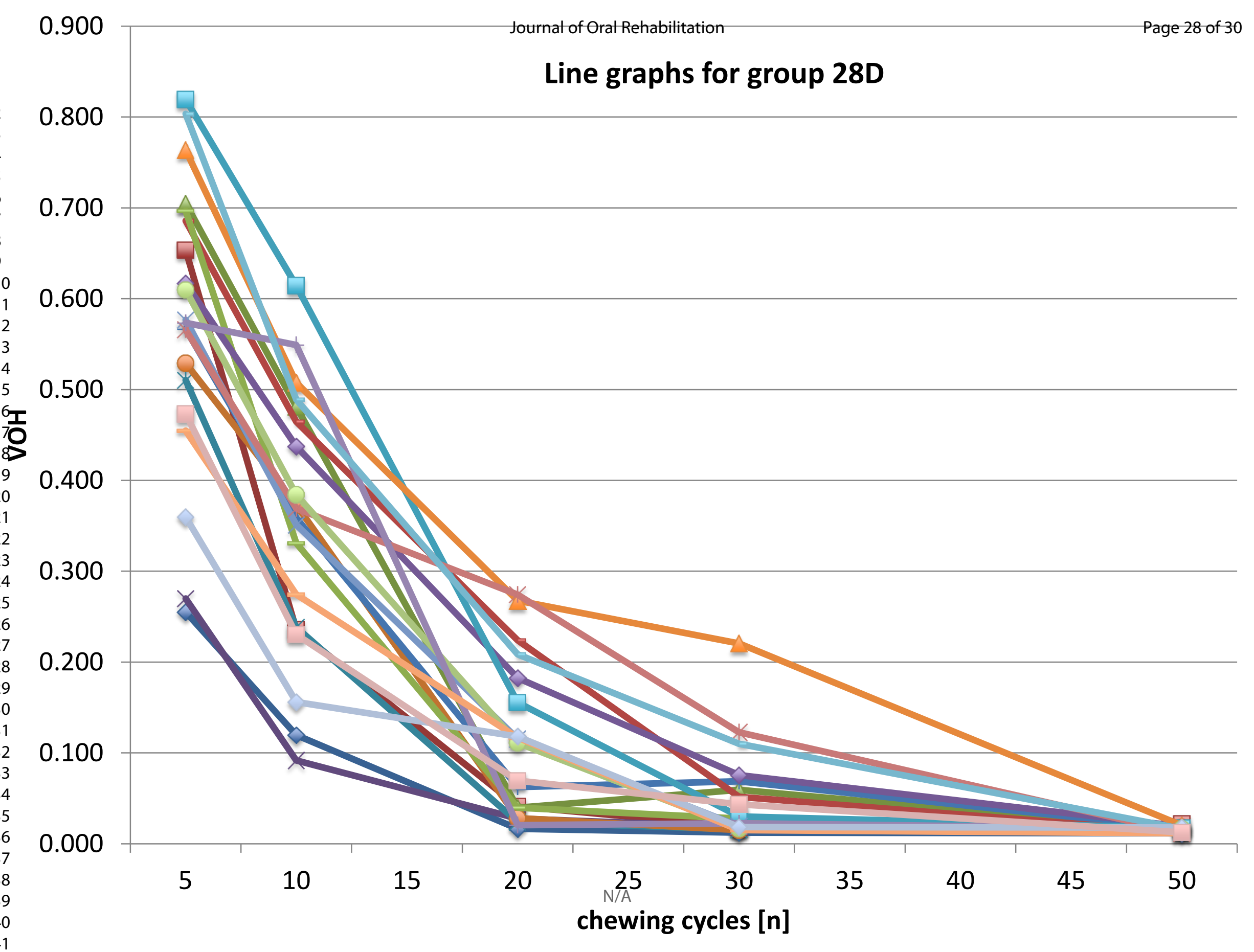


